\title{
An Ultracompact and Efficient Long-Wavelength Plasmonic Cut-Off Filter based on Asymmetric E-Shaped Resonator
}

\author{
Seyed Morteza Ebadi (1) *, Student Member, OSA, and Jonas Örtegren (1) \\ ${ }^{(1)}$ Department of Natural Sciences, Mid Sweden University, SE-85170 Sundsvall, Sweden \\ seyed-morteza.ebadi@miun.se
}

\begin{abstract}
This paper presents design and simulation results of a super-compact and efficient pla smonic cut-off filter based on an a symmetric E-shaped resonator. Moreover, by modulating length of the resonator, cut-off wavelength can be ea sily adjusted.
\end{abstract}

\section{Introduction}

Surface plasmon polaritons (SPPs), which are surface electromagnetic waves at a metal-dielectric interface at high frequencies coupled with free electron movement of the pla sma gas, give rise to guide and confinement of light beyond the diffraction limit, and enable integration of electronics and photonics on the same chip, which would lead to development of energy-efficient and miniaturized optical circuits. The plasmonic waveguide structure can be categorized as metal-insulator-metal (MIM) and insulator-metal-insulator (IMI), though, the first type is more attractive for inclusion in photonic integrated circuits (PICs), because of strong mode confinement and losses that are within acceptable levels [1 - 3]. Hence, researchers have proposed different components ba sed on the MIM waveguide like high-speed modulators [4], nanoscale refractive index sensing [5], light absorber [6], and so on. Some of them have been experimentally rea lized. Although optical filters play a significant role in a variety of applications including light detection and ranging (LIDAR), dispersion compensation, fluorescence microscopy, and spectroscopy, their development might have been overlooked. Accordingly, we report the design and numerical investiga tions of a highlycompact yet efficient tunable long-wavelength plasmonic cut-off filter based on MIM waveguide.

\section{Device Configuration and Simulation Results}
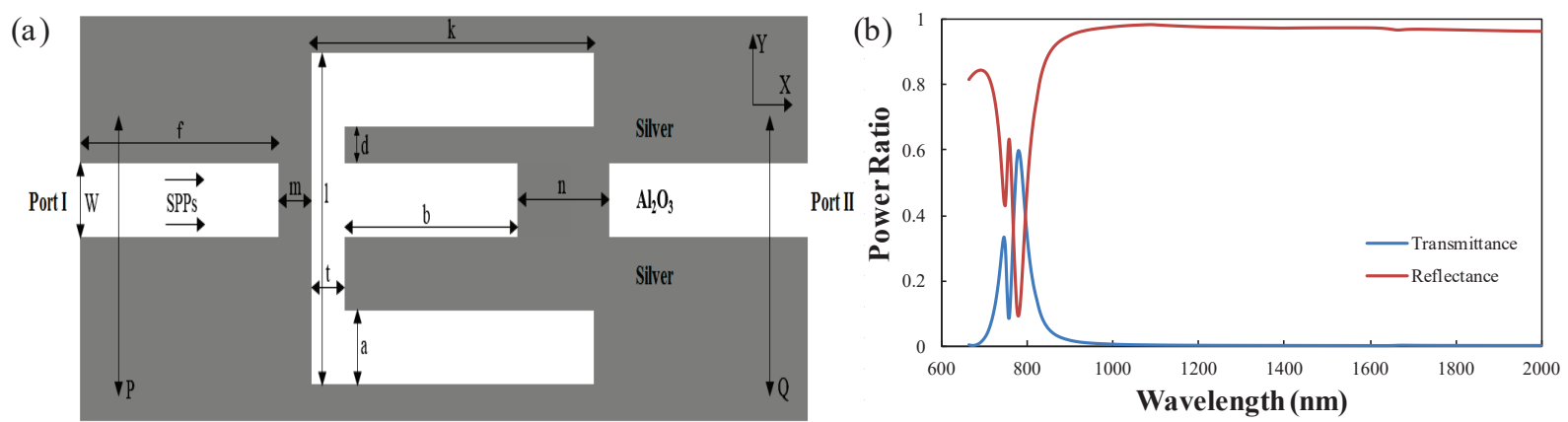

Fig. 1. (a) Schematic configuration of the suggested wide flat-top band-pass filter at NIR. (b) Transmittance and reflectance spectra of the wavelength filter for $\mathrm{W}=80 \mathrm{~nm}, \mathrm{~d}=40 \mathrm{~nm}, \mathrm{e}=160 \mathrm{~nm}, \mathrm{f}=\mathrm{b}=35 \mathrm{~nm}, \mathrm{a}=50 \mathrm{~nm}$, and $\mathrm{k}=185 \mathrm{~nm}$.

The new structure, which functions a s long-wavelength cut-off filter, is schematically demonstrated in Fig. 1. (a). The proposed device is composed of a layer of insulator that is set to be $\mathrm{Al}_{2} \mathrm{O}_{3}$, whose refractive index is found to be 1.75 [7], which is located between two metallic layers that a re made of silver, where the complex dielectric constant tabulated by Johnson and Christy [8] was used in the simulation. We have employed a full-wave EM software tool, CST MWS, with frequency domain solver that makes use of the finite element method (FEM) to achieve the numerical results presented in this paper [9]. The width of the input port is chosen much smaller than the wavelength of the incident light to ensure that only the fundamental TM mode propagates in the structure. A dipole source was utilized to excite the waveguide. The grid sizes are chosen to be $6 \mathrm{~nm} \times 6 \mathrm{~nm}$ along the $\mathrm{x}$ and $\mathrm{y}$ directions. To achieve the transmittance of the filter, two power monitors $\mathrm{P}$ and $\mathrm{Q}$ are located to capture the incident and transmitted light, from which follows that the transmittance can be described as T=Pout/Pin. The long-wavelength cut-off filter is obtained because of coupling between the bus waveguides and the asymmetric E-shaped resonator. The transmittance and reflectance spectra of the suggested cut-off filter is illustrated in Fig. 1. (b) for the structural parameters $\mathrm{W}=20 \mathrm{~nm}$, the width of the input port, $\mathrm{f}=80 \mathrm{~nm}$, the length of the bus waveguide, $\mathrm{m}=10 \mathrm{~nm}$, the coupling distance between the left waveguide and the 
resonator, $\mathrm{n}=30 \mathrm{~nm}$, the coupling distance from the right waveguide to the cavity. The sizes of the different segments of the E-shaped resonator are as follows; $\mathrm{l}=\mathrm{k}=90 \mathrm{~nm}$, as the length and width of the resonator, respectively, $\mathrm{d}=\mathrm{t}=10$ $\mathrm{nm}$, define the distance between the upper and middle tooth and the depth of the tooth, a=20 nm, shows the length of the tooth, and $b=60 \mathrm{~nm}$, as the width of the middle arm. The cut-off wavelength, defined as where the transmission reaches $1 \%$ [10], is revea led to be $928.6 \mathrm{~nm}$. It worth to mention that the ultra-wide cut-off occurs from the near-infrared (NIR) to the wavelength of $2 \mu \mathrm{m}$. In this wavelength interval, the transmission is constantly less than $1 \%$.

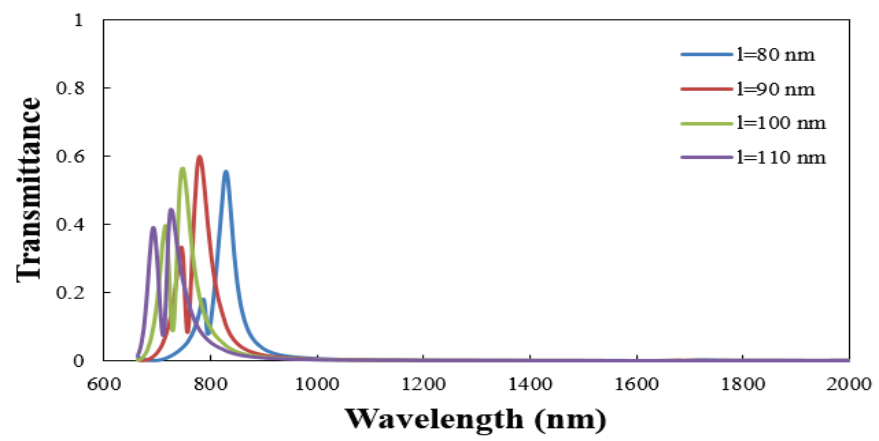

Fig. 2. The simulation results of the transmission profile for the long-wavelength cut-off filter for different values of the length of the resonator.

Figure 2 displays the simulation results of the suggested long-wavelength plasmonic cut-off wavelength filter for different lengths of the E-shaped resonator, whereas all other parameters are unchanged and have the same values as in Fig. 1. (a). The transmission spectra for $1=80 \mathrm{~nm}, 90 \mathrm{~nm}, 100 \mathrm{~nm}$, and $110 \mathrm{~nm}$, shown in Fig. 2, reveal that by reducing the value of the resonator length the cut-off wavelength is moved towards longer wavelengths, whereas by increasing the length of the E-shaped cavity, the cut-off wavelength moves to shorter wavelengths. In fact, for $1=80$ $\mathrm{nm}$, the cut-off wavelength is found to be $962.6 \mathrm{~nm}$, whilst for $1=110 \mathrm{~nm}$, it is $887.5 \mathrm{~nm}$. As a result, by simply choosing the appropriate length of the resonator, one can readily tune the cut-off wavelength of the structure.

\section{Conclusion}

In summary, a super-compact yet efficient tunable long-wavelength plasmonic cut-off filter is realized based on asymmetric E-shaped resonator. Numerical results confirm that tunability of the transmission profile can be realized through controlling the length of the resonator. Compared to recent studies [9-12], the new device offers smaller footprint and/orhigher efficiency, which makes it an attractive candidate in high-density on-chip integration.

\section{References}

[1] Ozbay, Ekmel. "Plasmonics: merging photonics and electronics at nanoscale dimensions." science 311.5758 (2006): $189-193$.

[2] Maier, Stefan Alexander. Plasmonics: fundamentals and applications. Springer Science \& Business Media, 2007.

[3] Pitarke, J. M., et al. "Theory of surfaceplasmons and surface-plasmon polaritons." Reports on progress in physics 70.1 (2006): 1.

[4] Hössbacher, Claudia, et al. "Plasmonic modulator with> 170 GHz bandwidth demonstrated at 100 GBd NRZ." Optics Express 25.3 (2017): $1762-1768$.

[5] Nishijima, Yoshiaki, et al. "Tailoring metal and insulator contributions in plasmonic perfect absorber metasurfaces." ACS Applied Nano Materials 1.7(2018): 3557-3564.

[6] Takatori, Kentaro, Takayuki Okamoto, and Koji Ishibashi. "Surface-plasmon-induced ultra-broadband light abs orber operating in the visible to infrared range." Optics express 26.2 (2018): 1342-1350.

[7] https://refractiveindex.info/?shelf=main\&book=Al2O3\&page=Malitson. (Accessed 10 March 2021).

[8] P. B. Johns on and R.-W. Christy, "Optical constants of the noble metals," Physical review B, vol. 6, no. 12, p. 4370, 1972.

[9] Ebadi, Seyed Morteza, et al. "High-efficiency nanoplasmonic wavelength filters based on MIM waveguides." IEEE Photonics Technology Letters 28.22 (2016): 2605-2608.

[10] Ebadi, Seyed Morteza, and Jonas Örtegren. "A Highly-Efficient and Compact Surface Plasmon Polaritons-based Long-Wavelength Cut-off Filter at Telecom Wavelengths." Novel Optical Materials and Applications. Optical Society of America, 2020.

[11] Zavvari, Mahdi, Milad Taleb Hesami Azar, and Armin Arashmehr. "Tunable band-stop plasmonic filter based on square ring res onators in a metal-insulator-metal structure." Journal of Modern Optics 64.20 (2017): 2221-2227.

[12] Pav, Mohammad Reza, et al. "Ultracompact double tunable two-channel plasmonic filter and 4-channel multi/demultiplexer design based on aperture-coupled plasmonic slot cavity." Optics Communications 437 (2019): 285-289. 